\title{
Bright lights in the big cities: migratory birds' exposure to artificial light
}

\author{
Kyle G Horton $^{1 \star}$, Cecilia Nilsson ${ }^{1}$, Benjamin M Van Doren ${ }^{2}$, Frank A La Sorte ${ }^{1}$, Adriaan M Dokter ${ }^{1}$, and Andrew Farnsworth ${ }^{1}$
}

Many species of migratory birds have evolved the ability to migrate at night, and the recent and rapid expansion of artificial light at night has markedly altered the nighttime sky through which they travel. Migrating birds regularly pass through heavily illuminated landscapes, and bright lights affect avian orientation. But risks to migrating birds from artificial light are not spatially or temporally uniform, representing a challenge for mitigating potential hazards and developing action plans to catalog risks at continental scales. We leveraged over two decades of remote-sensing data collected by weather surveillance radar and satellite-based sensors to identify locations and times of year when the highest numbers of migrating birds are exposed to light pollution in the contiguous US. Our continental-scale quantification of light exposure provides a novel opportunity for dynamic and targeted conservation strategies to address the hazards posed by light pollution to nocturnally migrating birds.

Front Ecol Environ 2019; 17(4):209-214, doi:10.1002/fee.2029

$\mathrm{T}$ rillions of flying organisms (eg birds, bats, insects) occupy the airspace within the troposphere during different periods of their annual cycles (Diehl 2013). The recent recognition of airspace as vital habitat - one that is subject to increasing modification by humans - highlights the fundamental need to understand how organisms cope with such alterations (Lambertucci et al. 2015), which pose numerous challenges to airborne organisms during periods of transit, including nocturnally migrating birds. Of the nearly 630 terrestrial species of birds regularly occurring in North America, approximately $70 \%$ are considered migratory, and of these more than $80 \%$ migrate at night (WebTable 1). Yet most studies of associated risks have focused on terrestrial habitats, underscoring a fundamental knowledge gap that can be addressed with recent technological (including computational) advances.

Light pollution of the airspace is a relatively recent but growing threat to nocturnally migrating birds (Longcore and Rich 2004; Van Doren et al. 2017; Cabrera-Cruz et al. 2018). Increasing urbanization has greatly amplified the amount of artificial light at night (ALAN; Kyba et al. 2017), with almost one-half of the contiguous US experiencing substantially photo-polluted nights (Falchi et al. 2016). Light sources including streetlights, safety lights, and extensively lit buildings - can disturb wildlife in a multitude of ways (Gauthreux and Belser 2005; Hölker et al. 2010; Rodríguez et al. 2017). Highpower light installations like lighthouses and communication towers are known to attract nocturnal migrants and are responsible for substantial mortality (Gauthreux and Belser 2005; Longcore et al. 2012). The numbers of birds attracted to or trapped by illumination depend on light wavelength (Poot

\footnotetext{
${ }^{1}$ Cornell Lab of Ornithology, Cornell University, Ithaca, NY *(kgh48@cornell.edu); ${ }^{2}$ Edward Grey Institute, Department of Zoology, University of Oxford, Oxford, UK
}

et al. 2008) as well as weather factors such as fog and precipitation (Gauthreux and Belser 2005). High-power light installations can even attract migrants in already heavily photopolluted areas and in skies with clear weather conditions (Van Doren et al. 2017).

An increasing number of artificial structures are now present in the lowest reaches of the troposphere (Davy et al. 2017), and their continued expansion poses an ever-increasing threat to wildlife. In the contiguous US, annual fatal bird collisions with buildings, communication towers, power lines, and wind turbines cumulatively number in the hundreds of millions (Loss et al. 2015). For nocturnally migrating birds, direct mortality as a result of collisions due to attraction to light (Gauthreux and Belser 2005) is the most obvious and direct effect of ALAN, but there are also more subtle effects, such as disrupted orientation (Poot et al. 2008) and changes in habitat selection (McLaren et al. 2018). There is also growing evidence that light pollution alters behavior at regional scales, with migrants occupying urban centers at higher-than-expected rates as a function of urban illumination (La Sorte et al. 2017). While ALAN acts as an attractant at both large (La Sorte et al. 2017) and local (Van Doren et al. 2017) scales, there is also evidence of migrating birds avoiding strongly lit areas when selecting critical resting sites needed to rebuild energy stores (McLaren et al. 2018).

\section{Challenges to conservation and mitigation}

To date, mitigating actions to reduce impacts of ALAN have involved directed and specific efforts, including reductions in excess lighting, the periodic switching off of highintensity lights (Van Doren et al. 2017), and adjusting wavelengths in situations where lights cannot be shut down (Poot et al. 2008; Longcore et al. 2018). These actions are 


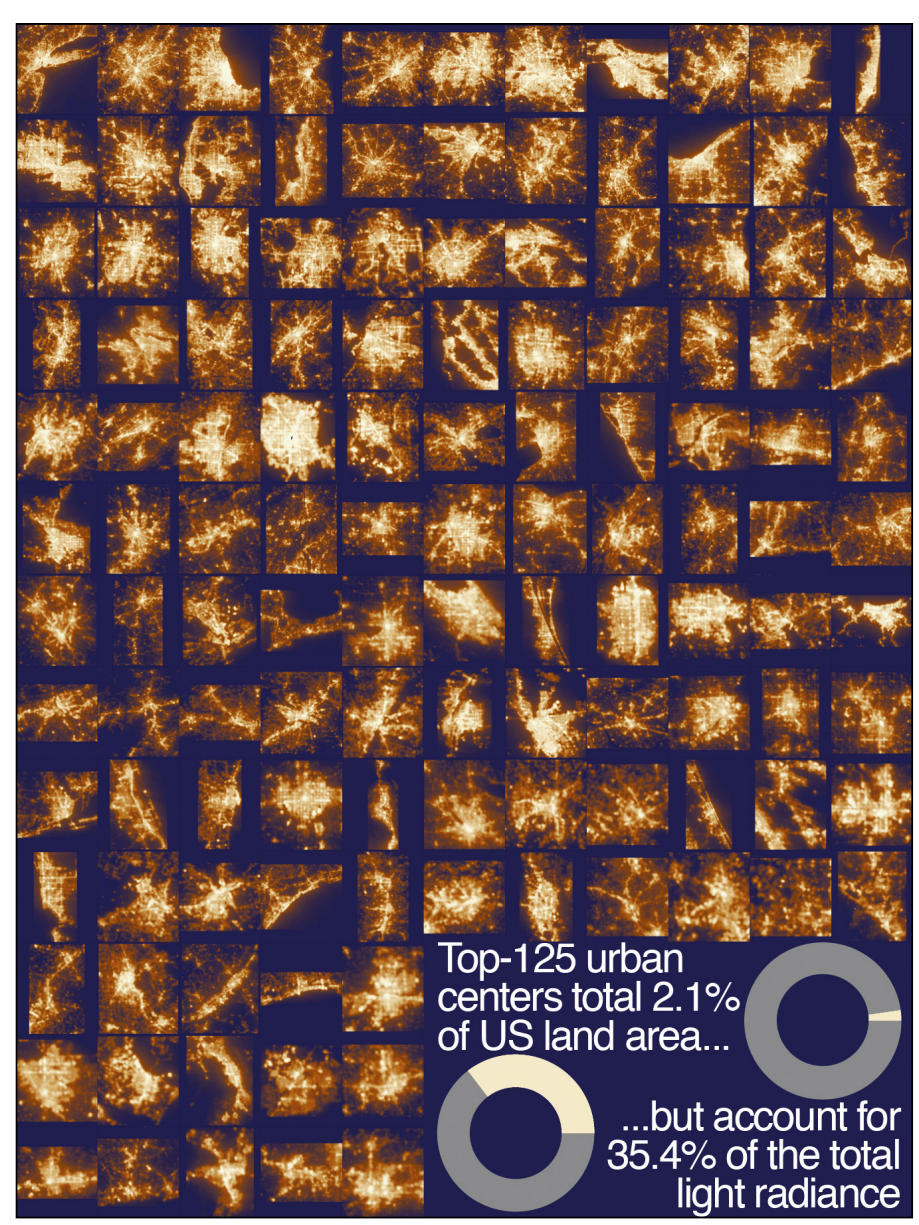

Figure 1. $\log _{10}$-scaled radiance of artificial light at night (ALAN) measured by the Visible Infrared Imaging Radiometer Suite (VIIRS) on the Suomi-NPP satellite of the 125 largest urban centers (by area) in the continental US. The largest urban center area is depicted in the top-left corner; the second largest area appears to its immediate right, and so forth, across the top-most row. In each successive row, urban center areas continue to decrease in size from left to right.

typically carried out at the scale of individual buildings but occasionally at much larger scales (eg Lights Out Toronto). However, as the intensity and extent of bird migrations vary considerably in space and over time (Van Doren and Horton 2018), so too may exposure risks, requiring detailed and site- and time-specific considerations when implementing mitigation actions and developing conservation plans. To this end, we used radar to quantify the passage of nocturnally migrating birds across the contiguous US, identified the areas where the greatest number of migrants are exposed to light pollution, and mapped this exposure across the US, focusing specifically on the 125 largest urban centers.

\section{Methods}

\section{Weather surveillance radar}

We used weather surveillance radar (WSR) data from 143 stations from spring (1 March to 31 May) and fall (15 August to 15 November) between spring 1995 and spring 2017 to characterize cumulative migration activity across the contiguous US. We acquired radar data through the Amazon Web Service portal, extracting data from a 30-minute window centered on 3 hours after local sunset. This time period was chosen because it represents the average peak in nocturnal migratory activity (eg Farnsworth et al. 2015; Horton et al. 2015; see Horton et al. [2018] and Van Doren and Horton [2018] for additional details regarding radar processing).

With respect to creating profiles of migration activity, we calculated altitude, speed, and direction using the lowest elevation scans $\left(0.5-4.5^{\circ}\right)$ at distances of $5 \mathrm{~km}$ to $37.5 \mathrm{~km}$ from the radar station (Farnsworth et al. 2015). We determined migration activity from reflectivity $\left(\eta, \mathrm{cm}^{2} \mathrm{~km}^{-3}\right)$ and flight direction and groundspeed from radial velocity between $100 \mathrm{~m}$ and $3000 \mathrm{~m}$ above ground level, at $100-\mathrm{m}$ altitudinal bins using the WSRLIB package (Sheldon 2015). We excluded altitudinal bins with velocity azimuth displays with root mean squared error (RMSE) $<1 \mathrm{~m} \mathrm{~s}^{-1}$ to limit contamination of radar readings by insects and removed samples with RMSE $>5 \mathrm{~m} \mathrm{~s}^{-1}$ to limit poor fits. In addition, we removed slow-flying objects (airspeed $<5 \mathrm{~m} \mathrm{~s}^{-1}$ ), which are representative of insects (Larkin 1991). To calculate airspeeds, we paired all radar measures of groundspeed and flight direction with wind measures using the North American Regional Reanalysis (NARR). Following these filtering procedures, we integrated reflectivity $\left(\mathrm{cm}^{2} \mathrm{~km}^{-3}\right)$ across the column of airspace sampled (100-3000 m) into vertically integrated reflectivity (VIR, $\mathrm{cm}^{2} \mathrm{~km}^{-2}$ ), which represented our measure of migration activity.

To discriminate contaminated scans (ie with precipitation) from precipitation-free scans (ie clear or biologically dominated), we designed a random forest classifier (Horton et al. 2019) using the R package "randomForest" (Liaw and Wiener 2002). We trained the classifier on 318,047 (spring: 157,279; fall: 160,768) manually classified nocturnal scans, selected from a 2.5 -hour period centered on 3 hours after local sunset on 15 March, 15 April, 15 May, 1 September, 1 October, and 1 November. Scans for each radar and for each year were represented in the training set. We extracted derived predictor variables from profiles of reflectivity, groundspeed, and summaries of the number of volumes above 35 decibels of reflectivity (dBZ) (a value typical of precipitation). We populated 1000 trees and restricted node size to 50 scans. The algorithm classified a total of 2,176,126 scans (spring: 979,326; fall: 1,196,800) with 5.6\% classification error during the spring and $4.5 \%$ during the fall, as determined using the manually classified scans. As an additional step to reduce the inclusion of samples classified as clear but containing weather, we used only scans with a confidence of being precipitation-free of $75 \%$ or higher (rather than a majority rule; ie $>50 \%$ ).

To extrapolate migration activity to areas not sampled by the radars, we relied on a generalized additive model using the $\mathrm{R}$ package "mgcv" (Wood 2011; R Core Team 2017). We first calculated the average migration activity for each ordinal day across all years and then summed each night through the sea- 
son to estimate the cumulative migration activity for each radar station. We fit radar station latitude, longitude, and the interaction of latitude and longitude with smoothing splines to predict the cumulative seasonal activity across the contiguous US.

\section{Artificial light at night}

We used the monthly Day/Night Band (DNB) product from the Visible Infrared Imaging Radiometer Suite (VIIRS) onboard the joint National Oceanic and Atmospheric Administration (NOAA)/National Aeronautics and Space Administration SuomiNPP satellite to quantify the magnitude of ALAN radiance (Earth Observation Group, NOAA National Geophysical Data Center; https://bit.ly/2nCjqvz). Monthly composites of radiance (nanoWatts per square centimeter per steradian; $\mathrm{nW} \mathrm{cm}^{-2}$ $\mathrm{sr}^{-1}$ ) are projected at 15 arc-second geographic resolution and are filtered to exclude data from stray light, lightning, lunar illumination, and cloud cover. However, because these data are not filtered for auroras and fires, we averaged across 3 months (October-December) over 6 years (2012-2017) to dampen the influence of episodic lighting events; these months were chosen because they fall outside the primary storm season in North America, which would obscure radiance measures. As an added step to ensure data quality, we excluded any pixel with fewer than 5 use-days prior to averaging monthly composites. Finally, we removed pixels with radiance values greater than $900 \mathrm{nW} \mathrm{cm} \mathrm{sr}^{-1}$ to remove wildfires and other ephemeral high-intensity lighting events (Kyba et al. 2017).

\section{Exposure index calculation}

To quantify migrant exposure to ALAN, we summarized exposure at two levels: (1) across the contiguous US and (2) in the top 125 largest urban centers by area (Figure 1). We used the 2017 US Census database to define the boundaries of these urban centers and used the primary city name in our presentation of urban area (eg Dallas-Fort Worth-Arlington, Texas, is presented as "Dallas"). Across the contiguous US, we calculated exposure as the product of cumulative migration activity $\times$ radiance, whereas over urban areas we calculated exposure as the product of cumulative migration activity $\times$ summed radiance of the entire urban area. To differentiate seasonal differences irrespective of increases in bird populations, we standardized cumulative migratory activity to range between 0 and 1 , and standardized activity relative to the highest value across the contiguous US. Exposure difference was calculated as the product of $\log _{10}$-scaled VIIRS radiance (standardized 0 to 1 ) and seasonal differences in migratory activity.

\section{Results}

\section{Migration activity}

Migration activity in spring was greatest in the central US (Figure 2a) and generally more widespread and more easterly

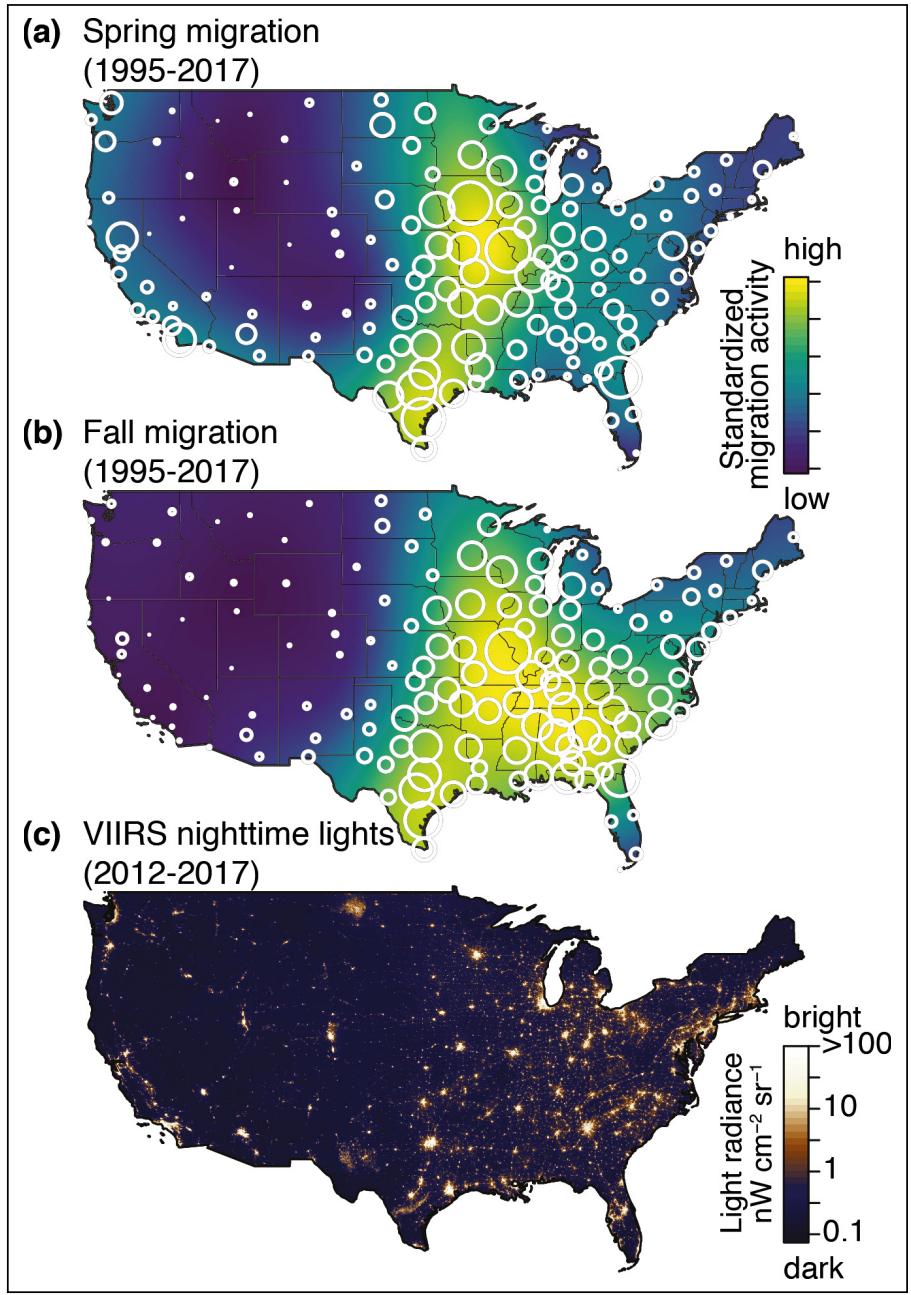

Figure 2. Average cumulative distribution of migrant birds during (a) spring and (b) fall migrations from 1995 to 2017 measured by weather surveillance radar (WSR). Circles indicate WSR station locations and are scaled to cumulative migration activity. The magnitudes of spring and fall cumulative movements are standardized to the same range. (c) $\log _{10^{-}}$ scaled mean radiance of ALAN measured by the VIIRS on the Suomi-NPP satellite.

in distribution in the fall (Figure 2b). In the western US, we observed greater migratory activity in the spring than in the fall. Furthermore, we observed a $63 \%$ increase in cumulative migratory activity from spring to fall. Examining the annual nightly pulses of migratory movements at each radar station, we observed that half of the cumulative migratory activity passed each station in $6.2 \pm 2.5$ (mean \pm standard deviation [SD]) nights in spring and $7.1 \pm 2.6$ nights in fall.

\section{Light pollution}

The general pattern of nightly radiance showed greater average radiance in the eastern half of the US, with a few notable exceptions from urban areas in the Pacific states, Desert Southwest, and a few Rocky Mountain cities (Figure 2c). As expected, the strongest radiance values were observed 


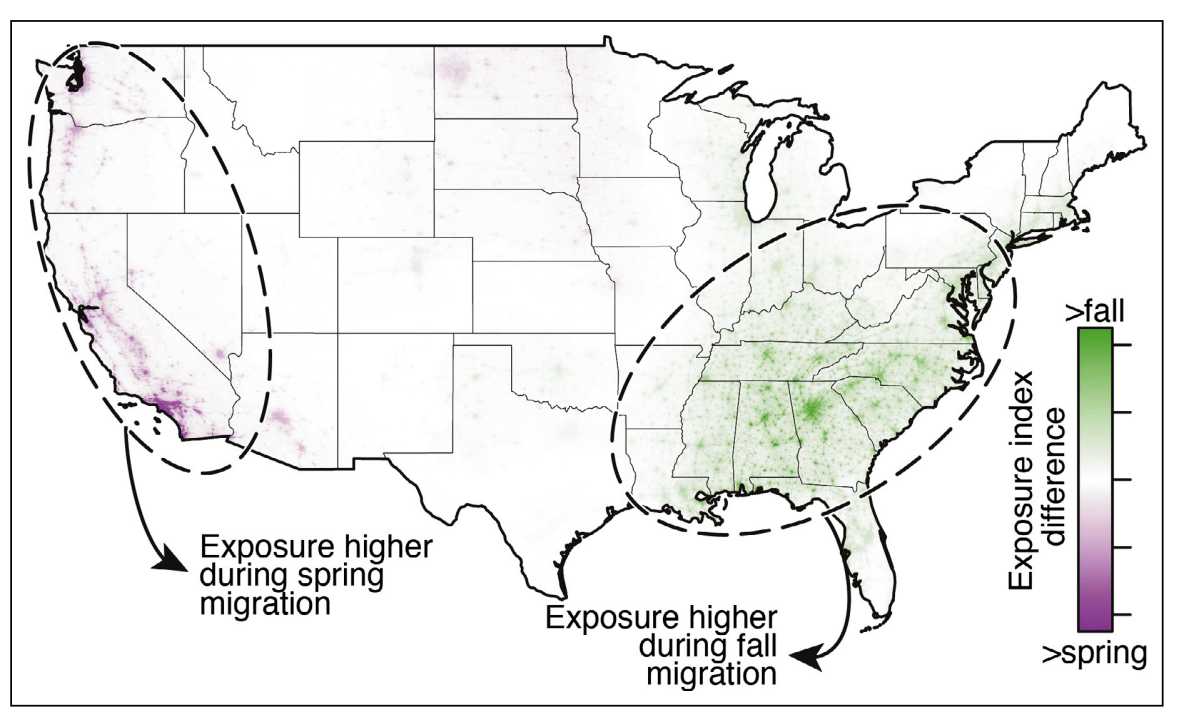

Figure 3. Seasonal differences in exposure to ALAN. The magnitude of spring and fall cumulative movements were standardized to the same range $\left(\begin{array}{l}0 \\ 0\end{array}\right.$ to 1$)$ to highlight seasonal differences in migratory routes. Exposure difference was calculated as the product of $\log _{10}$-scaled VIIRS radiance (standardized 0 to 1 ) and seasonal differences in migratory activity.

in urban areas. Across the US, $69.5 \%$ of the summed linear light radiance came from just $5 \%$ of the land area. The top 125 largest urban areas accounted for only $2.1 \%$ of total land area and $35.4 \%$ of total summed linear radiance.

\section{Continental exposure risk}

Increased migratory activity during fall was observed in almost all areas, resulting in $53.8 \%$ higher total of exposure in the fall. After standardizing for differences in overall migration activity between seasons, we determined that there was still a $13.1 \%$ higher sum of exposure in the fall, when migrants moved through more photo-polluted airspaces in the eastern half of the US (Figure 3). Departures from this trend were evident in the western half of the country, where spring movements along the Pacific coast led to higher spring exposure (Figure 3).

\section{Urban exposure risk over the 125 largest US cites}

Mean avian light exposure in cities was 24 times as high as the countrywide average. Larger cities tended toward greater exposure risk (linear regression, spring: $F_{1,123}=135.8, P<$ 0.001, $R^{2}=0.52$; fall: $F_{1,123}=203.1, P<0.001, R^{2}=0.62$ ), but there were notable exceptions, such as Boston (4th in size but 36th and 24th in exposure in spring and fall) and Des Moines (99th in size but 28th and 36th in exposure in spring and fall) (WebTable 2). Regardless of season, the highest levels of exposure to anthropogenic light at night were observed in Chicago, Houston, and Dallas, in descending order (Figure 4; see WebTable 2 for a complete list). These three cities showed exposure magnitudes that were 19 (spring) to 21 (fall) times as high as the median exposure of the remaining 122 cities (Figure 4a). In total, 45 and 74 urban areas exhibited higher spring exposure rankings and higher fall exposure rankings, respectively. Six areas, including Chicago, Houston, and Dallas, showed no change in ranking (Figure 4b). Of the 125 largest US cities, the top 10 greatest changes in seasonal rankings occurred in western states (eg Riverside, San Diego, San Jose; Figure $4 \mathrm{~b}$ ). Of the top 10 risks for exposure, the majority occurred in the central US: seven in spring and six in fall.

\section{Discussion}

We conducted a quantitative assessment of continent-scale exposure of actively migrating birds to nighttime light pollution. The findings leverage recent advances in data access and machine learning to capture new and rich details in characterizing bird movements aloft in relation to radiance from human population centers. With considerations for urban areas and the numbers of migrants flying above them, we can now provide the data necessary to guide conservation actions to identify locations where ALAN-reducing programs may be most effective.

\section{Shifted seasonal distributions}

Greater abundance of migrants in fall increases the number of birds at risk to ALAN, which was apparent in the general increase in exposure indices from spring to fall. However, shifts in migratory routes between spring and fall migration also affect the numbers of birds exposed to higher light levels (Figure 2). More easterly fall routes, often described as looped migration (La Sorte et al. 2014), take birds over more heavily photo-polluted areas than do spring routes, leading to even higher numbers of birds - and many young birds - exposed to ALAN in fall. At most sites, exposure indices are therefore higher during fall than in spring, indicating that any mitigation efforts (eg lights-out campaigns) would have a larger effect during the fall, especially with juveniles as they undertake their first migratory journey. However, while the risk of mortality for juveniles is likely to increase in the fall, any effects of ALAN on migrants in the spring will directly affect breeding activities. Birds moving along westerly routes during spring migration are the exception to this general pattern, likely related to their use of more westerly, low-elevation routes during spring as compared to fall (La Sorte et al. 2014). For example, the patterns in Los Angeles and other cities in California are the opposite of most East Coast cities, with higher exposure during spring migration (Figures 3 and 4).

\section{Uneven temporal distributions}

Migration periods may span more than 6 months in total, with hundreds of millions of individual migrants aloft on a 


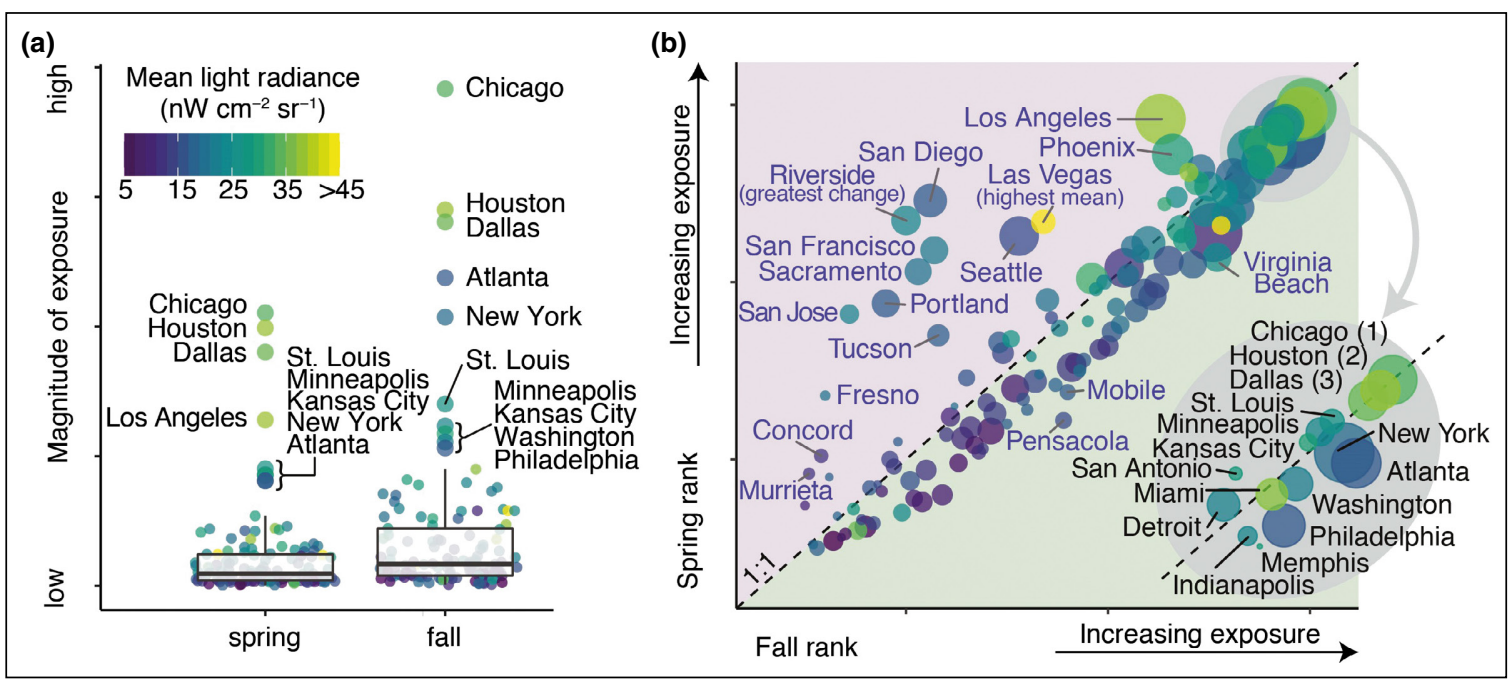

Figure 4. Seasonal (a) magnitude and (b) relative rankings of the 125 largest urban areas in the continental US. Point color shaded by the mean light radiance and sizes (in [b]) are scaled by the square root of urban area. (a) Only urban areas outside the 25th and 75th quartiles are labeled and (b) areas with a change in seasonal rank of $\geq 20$ positions are labeled and identified in blue. Inset in (b) depicts the top 15 (spring or fall) rankings; note that Los Angeles and Phoenix show both ranking changes $\geq 20$ and are ranked in the top 15, and therefore are not included in the inset. Urban areas in the purple shading (above the 1:1 dashed line) had higher spring exposure rankings, whereas those in the green shading (below the 1:1 dashed line) had higher fall exposure rankings.

given night; however, their passage occurs in sporadic waves, with a large majority of birds passing individual sites during just a few peak nights. We observed that half of the total number of migrants for each season passed each radar site in just $6.7 \pm 2.6$ (mean $\pm \mathrm{SD}$ ) nights, a notable finding when paired with the recent capacity to confidently forecast (12-72 hours in advance) these episodic events (Van Doren and Horton 2018). This advance has the potential to offer a detailed and tailored guide for mitigation actions to substantially lower the numbers of birds exposed to risks of ALAN while simultaneously minimizing adverse effects to stakeholders, including municipalities and industry. In addition, birds disproportionately use modified habitats (eg urban areas) during fall migration (Zuckerberg et al. 2016), and because migrants are more numerous and less experienced in fall, an emphasis on fall mitigation efforts is especially important.

\section{Conclusions}

ALAN continues to increase in many areas globally (Kyba et al. 2017), presenting an ever-growing ecological threat to all nocturnally active animals (Longcore and Rich 2004; Guetté et al. 2018), particularly migrating birds. Concerted conservation efforts at local (eg Van Doren et al. 2017) and continental scales are necessary to reduce exposure of migrants to light pollution. The disproportionate relationship between the land area occupied by cities and the amount of ALAN emitted leaves little doubt where conservation action is most needed: urban centers. Such efforts require balance with the needs of stakeholders. ALAN ranges from bright sources to dim stray light, and it remains an open question how conservation action should be prioritized over these widely differing sources. In addition, the extent to which species - or even populations - differentially respond to ALAN remains unclear, but could have important conservation implications. Furthermore, different datasets are available (eg a world atlas of artificial night sky brightness; Falchi et al. 2016), which may provide valuable information for characterizing ALAN's disruptions to aerial organismal biology (eg horizon glow versus upward radiance). Although we did not directly compare different sources of ALAN information with respect to exposure risks, we believe that such comparisons will be fundamentally important.

Reducing nighttime lights for the benefit of migrants and other wildlife represents yet another instance of anthropogenic and environmental trade-offs, in this case among avian safety, human safety, energy expenditure, and societal and psychological expectations. It is therefore important that conservation efforts and future research are directed to the times and places where they will have the largest impact. An important step in this direction is identifying where the highest numbers of birds are exposed to the highest amounts of ALAN. Here we have shown where the greatest threats exist, and how these threats vary seasonally. The combination of large amounts of nocturnal illumination and their location in the most trafficked airspace across the US elevate metropolitan Chicago, Houston, and Dallas to the top of the exposure risk ranking. While all urban areas should take care to minimize ALAN, our analysis indicates that actions taken in these particular cities would benefit the largest numbers of birds. Through our analysis, we have identified risk; however, directly linking risk with adverse effects on bird populations is a challenge, and future research is needed to fully understand the impacts of ALAN on migratory species. 


\section{Acknowledgements}

We thank S Kelling for constructive feedback on early drafts of this manuscript. This work was supported by three Edward W Rose Postdoctoral Fellowships, Marshall Aid Commemoration Commission, Leon Levy Foundation, Wolf Creek Charitable Foundation, and US National Science Foundation IIS-1633206 and DBI-1661329.

\section{References}

Cabrera-Cruz SA, Smolinsky JA, and Buler JJ. 2018. Light pollution is greatest within migration passage areas for nocturnally-migrating birds around the world. Sci Rep-UK 8: 3261.

Davy CM, Ford AT, and Fraser KC. 2017. Aeroconservation for the fragmented skies. Conserv Lett 10: 773-80.

Diehl RH. 2013. The airspace is habitat. Trends Ecol Evol 28: 377-79.

Falchi F, Cinzano P, Duriscoe D, et al. 2016. The new world atlas of artificial night sky brightness. Science Advances 2: e1600377.

Farnsworth A, Van Doren BM, Hochachka WM, et al. 2015. A characterization of autumn nocturnal migration detected by weather surveillance radars in the northeastern US. Ecol Appl 26: 752-70.

Gauthreux SA and Belser CG. 2005. Effects of artificial night lighting on migrating birds. In: Rich C and Longcore $\mathrm{T}$ (Eds). Ecological consequences of artificial night lighting. Washington, DC: Island Press.

Guetté A, Godet L, Juigner M, and Robin M. 2018. Worldwide increase in artificial light at night around protected areas and within biodiversity hotspots. Biol Conserv 223: 97-103.

Hölker F, Wolter C, Perkin EK, and Tockner K. 2010. Light pollution as a biodiversity threat. Trends Ecol Evol 25: 681-82.

Horton KG, Shriver WG, and Buler JJ. 2015. A comparison of traffic estimates of nocturnal flying animals using radar, thermal imaging, and acoustic recording. Ecol Appl 25: 390-401.

Horton KG, Van Doren BM, La Sorte FA, et al. 2018. Navigating north: how body mass and winds shape avian flight behaviours across a North American migratory flyway. Ecol Lett 21: 1055-64.

Horton KG, Van Doren BM, La Sorte FA, et al. 2019. Holding steady: little change in intensity or timing of bird migration over the Gulf of Mexico. Glob Change Biol 25: 1106-18.

Kyba CCM, Kuester T, de Miguel AS, et al. 2017. Artificially lit surface of Earth at night increasing in radiance and extent. Science Advances 3: e1701528.

La Sorte FA, Fink D, Buler JJ, et al. 2017. Seasonal associations with urban light pollution for nocturnally migrating bird populations. Glob Change Biol 23: 4609-19.

La Sorte FA, Fink D, Hochachka WM, et al. 2014. Spring phenology of ecological productivity contributes to the use of looped migration strategies by birds. P Roy Soc B-Biol Sci 281: 20140984.
Lambertucci SA, Shepard ELC, and Wilson RP. 2015. Human-wildlife conflicts in a crowded airspace. Science 348: 502-04.

Larkin RP. 1991. Flight speeds observed with radar, a correction: slow "birds" are insects. Behav Ecol Sociobiol 29: 221-24.

Liaw A and Wiener M. 2002. Classification and regression by randomForest. $R$ News 2: 18-22.

Longcore T and Rich C. 2004. Ecological light pollution. Front Ecol Environ 2: 191-98.

Longcore T, Rich C, Mineau P, et al. 2012. An estimate of avian mortality at communication towers in the United States and Canada. PLoS ONE 7: e34025.

Longcore T, Rodríguez A, Witherington B, et al. 2018. Rapid assessment of lamp spectrum to quantify ecological effects of light at night. J Exp Zool Part A 329: 511-21.

Loss SR, Will T, and Marra PP. 2015. Direct mortality of birds from anthropogenic causes. Annu Rev Ecol Evol S 46: 99-120.

McLaren JD, Buler JJ, Schreckengost T, et al. 2018. Artificial light at night confounds broad-scale habitat use by migrating birds. Ecol Lett 21: 356-64.

Poot H, Ens BJ, De Vries H, et al. 2008. Green light for nocturnally migrating birds. Ecol Soc 13: 47.

R Core Team. 2017. R: a language and environment for statistical computing. Vienna, Austria: R Foundation for Statistical Computing.

Rodríguez A, Holmes ND, Ryan PG, et al. 2017. Seabird mortality induced by land-based artificial lights. Conserv Biol 31: 986-1001.

Sheldon D. 2015. WSRLIB: MATLAB toolbox for weather surveillance radar. Amherst, MA: School of Computing, University of Massachusetts.

Van Doren BM and Horton KG. 2018. A continental system for forecasting bird migration. Science 361: 1115-18.

Van Doren BM, Horton KG, Dokter AM, et al. 2017. High-intensity urban light installation dramatically alters nocturnal bird migration. P Natl Acad Sci USA 114: 11175-80.

Wood SN. 2011. Fast stable restricted maximum likelihood and marginal likelihood estimation of semiparametric generalized linear models. J Roy Stat Soc B 73: 3-36.

Zuckerberg B, Fink D, La Sorte F, et al. 2016. Novel seasonal land cover associations for eastern North American forest birds identified through dynamic species distribution modelling. Divers Distrib 22: 717-30.

\section{Supporting Information}

Additional, web-only material may be found in the online version of this article at http://onlinelibrary.wiley.com/doi/ 10.1002/fee.2029/suppinfo 